\title{
Amour et mort, vie de sainteté dans la littérature et la spiritualité à València au XVème siècle: de saint Vicent Ferrer à Sor Isabel de Villena
}

\author{
Love and death, life of holiness in literature and spirituality \\ in Valencia in the 15th century: from Saint Vicent Ferrer to Sor Isabel de \\ Villena
}

Dominique De Courcelles dominique.decourcelles@club-internet.fr

Paris Sciences Lettres Université de la Recherche (CNRS-Ecole normale supérieure Ulm)

Institut d'Estudis Catalans-Reial Acadèmia de Bones Lletres de Barcelona

\begin{abstract}
Résumé: Le Royaume de València, au XVème siècle, tient une place majeure dans l'histoire de la littérature et de la spiritualité et dans l'histoire religieuse de la péninsule Ibérique, cependant que s'achève la Reconquista et que s'unifient les Espagnes. Une même quête de réforme morale et d'élévation spirituelle s'exprime aussi bien dans les sermons du dominicain Fra Vicent Ferrer que dans les traductions d'auteurs classiques et de la Renaissance effectuées par un autre dominicain, Fra Antoni Canals. La spiritualité valencienne est militante : elle veut convaincre, séduire, donner et parfois imposer ce qu'elle reconnait comme indispensable à la perfection et au salut. Les héros valenciens du XVème siècle sont des chevaliers et des religieux, des saints et des saintes. Ils manient l'épée ou la parole, ou les deux, pour défendre les valeurs du christianisme, l'art d'aimer et de mourir chrétiennement, la vérité, la justice, la paix. C'est ainsi que l'anonyme roman de Curial e Güelfa ou le Cant espiritual du chevalier Ausiàs March témoignent de la jonction entre chevalerie et théologie, cependant que Joanot Martorell, chevalier auteur du roman de Tirant lo Blanc, et Sor Isabel de Villena, abbesse des clarisses de la Trinité, auteur d'une Vita Christi, désignent, chacun à sa mesure, une ouverture spirituelle de l'histoire, une nécessaire conversion intérieure. Des joutes poétiques et théologiques réunissent clercs et chevaliers, entrelaçant sainteté et amour sensible et permettant l'élaboration des goigs, célèbres prières chantées qui prendront toute leur importance dans la vie spirituelle des fidèles après le concile de Trente.
\end{abstract}

Mots clef: Chevalerie, Joutes, poétiques, Littérature, Spiritualité, Théologie

\begin{abstract}
The Kingdom of Valencia, in the 15th century, holds a major place in the history of literature and spirituality and in the religious history of the Iberian Peninsula, while the Reconquista is completed and the Spanish are united. The same quest for moral reform and spiritual elevation is expressed in the sermons of the Dominican fra Vicent Ferrer as well as in the translations of classical and Renaissance writers by another Dominican, Fra Antoni Canals. Valencian spirituality is militant: it wants to convince, seduce, give and sometimes impose what it recognizes as indispensable to perfection and salvation. Valencian heroes of the 15th century are knights and religious and saints. They wield the sword or the word, or both, to defend the values of Christianity, the art of loving and dying Christianity, truth, justice, peace. Thus the anonymous novel by Curial e Güelfa or the Cant espiritual of the knight Ausiàs March testify to the junction between chivalry and theology, while Joanot Martorell, knight author of the novel of Tirant lo Blanc, and Sor Isabel de Villena, Abbess of the clares of the Trinity, author of a Vita Christi, designate, each to its own measure, a spiritual opening of history, a necessary interior conversion. Poetic and theological games bring together clergy and knights, intertwining holiness and sensitive love and allowing the development of goigs, famous sung prayers that will take all their importance in the spiritual life of the faithful after the Council of Trent.
\end{abstract}

Keywords: Chivalry ,Poetic, Jousting, Literature, Spirituality, Theology. 
Il y a des lieux où souffle l'esprit. València au XVème siècle est un de ces lieux. La vie sociale et culturelle, politique, religieuse y est alors profondément marquée par la guerre dite de Reconquista qui dure depuis des siècles et qui a trouvé son aboutissement dans la conquête ou reconquête des terres de València par le roi Jaume I d'Aragon « el Conquistador » en 1238. Avec les Furs de València, privilèges de la population d'origine, dont la rédaction est sanctionnée par le roi, un royaume est fondé avec son identité politique propre, ses institutions, sa culture et ses traditions, sa monnaie, sa langue, son administration, tout en étant intégré dans la royauté aragonaise. La rechristianisation des villes et des villages est alors un enjeu déterminant pour les pouvoirs politiques et ecclésiastiques; la religion chrétienne veut s'avérer créatrice de culture et de vie nouvelle pour l'unification d'une royauté chrétienne, catholique, aragonaise, cependant qu'un certain scepticisme tend à se répandre tout au long du XIVème siècle avec le nominalisme de Guillaume d'Ockham (1290-1349) et surtout avec le Grand Schisme (1378-1417). Le Royaume de València est alors peuplé de musulmans, de juifs et de chrétiens venus du nord et surtout installés près des côtes pour peupler chrétiennement le Royaume.

Le XVème siècle est une époque de changements profonds. Les monarques achèvent la conquête et l'unification des Espagnes et doivent souvent lutter contre les grands féodaux de leurs États. Les visions politiques, sociales et religieuses du monde changent. Dans ce contexte, le Royaume de València, reconquis et unifié alors que s'achève la Reconquista de la péninsule Ibérique, est le point de départ de l'aventure italienne du nouveau roi d'Aragon, Alphonse le Magnanime, à Naples et aussi en Sardaigne, Corse, Sicile et Djerba ; c'est la pieuse reine Maria de Castille qui exerce en l'absence du roi la lieutenance générale de ses Etats d'Aragon et Catalogne, et elle se plaît à résider à València (où elle meurt en 1458). Le Royaume de València s'affirme alors comme le grand défenseur et diffuseur de la foi catholique et l'Eglise contribue à son dynamisme et à son expansion politique. La spiritualité valencienne est militante, c'est-à-dire qu'elle veut convaincre, séduire, donner et parfois imposer ce qu'elle reconnait comme indispensable à la perfection humaine et au salut éternel. Les héros valenciens du XVème siècle sont des chevaliers et des religieux, des saints et des saintes. Ils manient l'épée ou le verbe sacré, ou les deux, pour défendre les grandes valeurs spirituelles du christianisme, l'art d'aimer et de mourir chrétiennement, la vérité, la justice, la paix.

\section{Prédication et traductions}

Dans ce contexte, l'art de la prédication connaît nécessairement à València un très grand développement. Au début du XVème siècle, le grand prédicateur est le dominicain Fra Vicent Ferrer, né à València en 1350. Savant maitre en théologie, il est surtout un magnifique orateur, redoutable pourfendeur du péché et de l'hérésie, prêchant la pénitence, désireux de convertir juifs et maures et contribuant ainsi, comme les chevaliers chrétiens, à l'unification chrétienne de la péninsule Ibérique et au salut des âmes dans la perspective du jour du jugement. Il meurt en 1419 
Dominique de Courcelles. Amour et mort, vie de sainteté dans la littérature et la spiritualité à València au XVème siècle: de saint Vicent Ferrer à Sor Isabel de Villena

à Vannes, et c'est un pape valencien, Calixte III, qui le canonise en 1455. Un retable provenant du couvent de Santo Domingo de València le représente prêchant dans une église, debout et dominant un public attentif d'hommes et de femmes, parmi lesquels deux scribes notent ses paroles (Museu de Belles Artes de Sant Carles, València). Il est significatif que de nombreux sermons de Fra Vicent Ferrer évoquent des situations guerrières. Josep Antoni Aguilar en a donné de très beaux exemples. Je citerai encore:

Si hun rey havia hun castell e hun enemich lo li escalava, al rey mal li sabría de aquell quant o sabría, mas no s’i daría molt. Mas si hun cavaller seu lo hi escalava, pus mal li sabría, per què com aquel és cavaller, domèstich seu, e l'altre és enemich. Sus així és: hun moro o altre infel fa desplaer a Déu, mal li sap; mas tu, christià, li fas més desplaer, e per ço lo juheu e·l moro será dapnat, mas més dapnat seràs tu,christià, qui tens mala vida. ${ }^{1}$

L'exemple donné par le prédicateur est particulièrement efficace, dans la mesure où l'auditoire est évidemment très averti des problèmes de châteaux pris par des ennemis ou par des traitres. Le baptême, s'il est sincère et véritable, doit amener à l'amour de Dieu et à la fidélité qui est aussi la qualité par excellence du chevalier envers son suzerain. Amour et fidélité, gages d'une vie chrétienne et spirituelle, sont ici les conditions de l'accès au paradis après la mort.

En ce même début du XVème siècle valencien, l'amour des livres et les traductions d'auteurs classiques ou de la Renaissance participent de la même quête de réforme morale et d'élévation spirituelle, qui est exprimée dans les sermons de Fra Vicent Ferrer, et sont bien susceptibles d'amener les valenciens à de nouvelles préoccupations morales et spirituelles. Fra Antoni Canals qui naît dans le Royaume de València vers 1352, lui aussi dominicain, meurt comme Vicent Ferrer en 1419. S'il traduit les grands auteurs classiques pour leur sens moral, en particulier Valère Maxime et Sénèque, il traduit également le mystique Hugues de Saint-Victor, en langue vernaculaire. Dans son prologue à la traduction de la lettre De modo bene vivendi faussement attribuée à saint Bernard (effectuée entre 1396 et 1410), qu'il adresse à Galceran de Sentmenat, camerlenc du roi Martin l'Humain, il déclare:

En los libres Déu, qui és incomprensible, aprensiblement és contengut. En los libres trop jo
los morts així com si eren vius ; en los libres jutge de les coses esdevenidores, en los libres són
dispostes les batalles, dels libres ixen los drets de pau. Totes les coses jusanes se corrompen e
cessen, e la mort continuadament devora aquells los quals natura engendra. Tota la glòria del
món seria lliurada a l'oblit si Déu no hagués provesit als hòmens mortals del benefici dels libres.

Sénèque, saint Bernard et Hugues de Saint-Victor sont les auteurs qui inspirent en la fin du XIVème siècle la « devotio moderna » naissante pour une pratique religieuse plus personnelle, fondée sur la méditation et l'amour de Dieu et du prochain.

1 Sermó del dijous 4 de juny de 1416, predicat a Castras: Sermó IX, dans Sant Vicent Ferrer (1932: 100).

2Edició de la Carta de sant Bernat a sa germana (CDIACA, XIII, 1857: 416-417).

SCRIPTA, Revista internacional de literatura i cultura medieval i moderna, núm. 14/ desembre 2019/ pp. 276 - 288 ISSN: 2340-4841 ·doi:10.7203/SCRIPTA.14.16370 
La beauté de la prose de la traduction du Soliloquium de arrba animae de Hugues de Saint-Victor, comme l'ont bien soulignée les critiques, montre que Fra Antoni Canals est avant tout un grand spirituel, ce qui s'accorde bien avec la valeur mystique de sa Scala de Contemplació. Cette ouvre se partage en trois livres. Dans le prologue adressé au roi Martí, Fra Antoni Canals explique :

\begin{abstract}
En lo primer se mostra com puia nostra ànima per la dita scala, e çò per contemplació de les coses iusanes, e fenex a les penes de infern. En lo segon se mostra com puia la dita ànima per la dita scala per contemplació dels òrdens del cel e de la glòria de paradís, de on devallà lo Fill de Déu per nostra redemció; e fenex aquest libre a la contemplació de la passió de Thesu Crist. En lo tercer se mostra com puia la dita ànima per la dita scala per contemplació de les dolçós de paradís e per los goigs dels sancts a la visió del Creador (1975: 60).
\end{abstract}

Il ne manque pas de rappeler ensuite que le roi a voulu fonder la chartreuse de Vall de Jesucrist, alors sur le point d'être consacrée, et que ce qui importe avec cette fondation ce sont les «libres de spiritual intel-ligèntia » offerts à la méditation des religieux.

Ainsi le dominicain Fra Antoni Canals contribue de façon tout à fait remarquable à l'essor de l'histoire intellectuelle et spirituelle de la València du XVème siècle. València au XVème siècle est en effet un des centres majeurs de la Renaissance européenne par les traductions qui s’y développent en langue vernaculaire valencienne d'œuvres latines classiques de morale et de philosophie, de littérature, d'histoire. València est aussi un lieu de création artistique, littéraire, philosophique, spirituel, où se marquent les principes de la « devotio moderna ». La richesse et la beauté de la langue valencienne renforcées par les traductions de la langue latine accompagnent la richesse et la beauté des créations d'architecture, de peinture, de musique du Royaume de València.

\title{
2. Chevalerie et théologie
}

Dans l'énigmatique roman chevaleresque Curiale Güelfa, écrit au XVème siècle, c'est grâce à Melchior de Pandone, humaniste stö̈cien sur fond de platonisme et de christianisme, que se réalise la difficile conciliation ou coïncidence des opposés entre, d'une part, la courtoisie, l'élégance et la force qui caractérisent la chevalerie idéale, et d'autre part, la puissance religieuse et chrétienne. Le chevalier Curial, de courtisan qu'il était, devient maître de lui-même et capable de s'adjoindre cette puissance religieuse et chrétienne, qu'il a intériorisée, grâce à une morale stoïcienne de perfectionnement moral qui a su préserver la supériorité du platonisme et, en fin de compte, du christianisme. Dans la ligne d'auteurs classiques comme Valère Maxime, Sénèque et Macrobe, Melchior de Pandone représente ainsi la voie de philosophes et de poètes comme Marsile Ficin, Politien, Pic de la Mirandole, qui sont ses contemporains en Italie (De Courcelles, 2012: 243-252).

Le chevalier poète Ausiàs March (vers 1397-1459), si aimé du roi Alphonse le Magnanime qu’il a accompagné en Italie, par ailleurs si dur et querelleur dans la gestion de ses propriétés de Beniarjó, 


\title{
Dominique de Courcelles. Amour et mort, vie de sainteté dans la littérature et la spiritualité à València au XVème siècle: de saint Vicent Ferrer à Sor Isabel de Villena
}

Pardines et Verniçà dans le duché de Gandia, est bien un de ces chevaliers du Royaume de València sans doute nostalgiques des temps de la Reconquista, qui ne cessent d'échanger des lettres de bataille et de se battre entre eux lorsqu'ils ne sont pas en aventure. Mais Ausiàs March est aussi très savant, épris de poésie, de théologie et de philosophie morale, auteur d'une œuvre poétique importante et célèbre. Ainsi, dans le Cant espiritual, qui est une longue prière adressée à Dieu en 224 vers, il se reconnait pécheur et incapable d'aller vers Dieu, alors que son libre arbitre le souhaite:

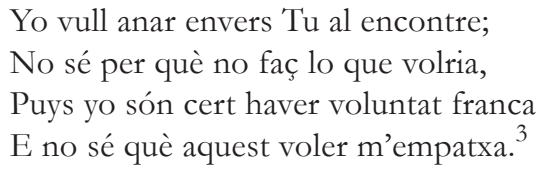

C'est une «terrible batalla » qui se livre dans l'âme du chevalier. Mais ce qu'il veut -sa « voluntat franca », c'est aimer Dieu, pour échapper à la damnation que lui valent ses fautes. Peu importent les dons « de natura i fortuna », habituellement demandés à Dieu dans les prières traditionnelles.

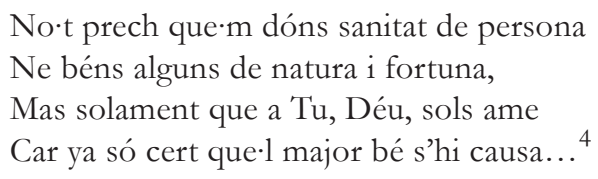

Ausiàs March sait que, sans amour pour Dieu, on ne peut entrer dans la gloire divine. Il a l'espoir que cet amour, difficile, peut lui être accordé et il désire alors la mort, tout en la redoutant, afin de ne plus avoir la tentation de pécher:

\footnotetext{
Qual serà·l jorn que la mort yo ne tema?

E será quant de t'amor yo·m inflame,

E no.s pot fer sens menyspreu de la vida,

E que per Tu aquella jo menyspree. ${ }^{5}$
}

Le mépris de la vie est bien cette insensibilité aux plaisirs et délices qui conditionne le vif et enflammé amour pour Dieu. Le chevalier poète en arrive alors à poser la question, si présente et angoissante pour les hommes et les femmes du XVème siècle, de la prédestination :

\footnotetext{
Tu creist mé perquè l'ànima salve

E pot-se fer de mi sabs lo contrari.

Si és així, per què, donchs, me creaves,

Puix fon en tu lo saber infal-lible?
}

\author{
3 March (ed. 1990: 126), Poema CV, Cant Espiritual, vv. 4-8. \\ 4March (ed. 1990: 136), Poema CV, Cant Espiritual (vv. 153-156). \\ 5March (ed. 1990: 136), Poema CV, Cant Espiritual (vv. 161-164).
}

SCRIPTA, Revista internacional de literatura i cultura medieval i moderna, núm. 14/ desembre 2019/ pp. 276 - 288 


\section{Dominique de Courcelles. Amour et mort, vie de sainteté dans la littérature et la spiritualité à València au XVème siècle: de saint Vicent Ferrer à Sor Isabel de Villena}

Torn a no-res, yo·t suplich, lo meu ésser,

Car més me val que tostemps l'escur càrcer. ${ }^{6}$

Le non-être vaut mieux que l'enfer. C'est ainsi que le chevalier poète ne doute pas de la justice de Dieu et de l'éternité de l'homme pour le meilleur ou pour le pire.

\section{Chevalerie morale et renoncement à l'Orient}

Joanot Martorell (vers 1413/1414-1468), chevalier valencien, dont la sœur Isabel a été l'épouse d'Ausiàs March pendant deux ans avant de mourir et dont le frère a échangé des lettres de bataille avec Ausiàs March, est lui-même bien représentatif de la petite noblesse valencienne du XVème siècle. Respecté pour sa maitrise des armes, il passe sa vie en combats singuliers et en voyages dans des cours lointaines, en particulier en Angleterre. Il est également très savant, grand lecteur de littérature et attentif aux bibliothèques qu'il découvre au cours de ses déplacements.

Joanot Martorell a la conviction que c'est l'art de chevalerie qui peut sauver la chrétienté et le monde; ce sont les valeurs de la chevalerie qui permettent de vivre, d'aimer et de mourir dans la perspective de la rédemption du monde, de tous les êtres humains et de soi. Il commence le 2 janvier 1460 son roman Tirant lo Blanc, imprimé en 1490 après sa mort, par l'initiation de son héros Tirant à l'Ordre de chevalerie par un chevalier, Guillem de Varoic, «le meilleur chevalier du monde ", devenu ermite au seul service de Dieu, après s'être séparé de son épouse. Le valencien Joanot Martorell trouve ici son inspiration dans un célèbre ouvrage de Ramon Llull, le Libre de l'orde de cavalleria, et l'on sait que le lullisme est important à València et donnera lieu à la publication à València, en 1521, chez Johan Joffre, du grand roman de Ramon Llull, Blanquerna $^{7}$. Il trouve également son inspiration dans un roman anglo-normand du XIIIème siècle, Guy de Warwycke, adapté en prose française, qu'il a peut-être lu à Londres. Le roman Tirant lo Blanc, nous dit son auteur, est lui-même la traduction «en vulgar llengua valenciana » d'un livre « anglés » traduit « en llengua portuguesa ». Joanot Martorell trouve aussi son inspiration dans le célèbre Arbre des Batailles d'Honoré Bouvet, dont le chevalier ermite lit un passage à Tirant:

Fallint en lo món caritat, lealtat e veritat, començá mala voluntat, injúria e falcedat, e per ço fon gran error entre lo poble de Déu, e gran confusió. E per ço que Deu sia amat, conegut, honrat, servit e tenut en lo món, en lo principi fonc poc stimada justícia per defalliment de caritat, per què fon necessària cosa e condecent que justícia fos tornada en sa honor e prosperitat.

E, per aquesta causa, de tot lo poble foren fets mil·lenars, e de cascun mil·ler fonch elet un

6 March (ed. 1990: 138), Poema CV, Cant Espiritual (vv. 193-198).

7 «Traduit i corregit ara novament dels primers originals, y estampat en lengua Valenciana» par Joan Bonllavi, célèbre lulliste de València.

SCRIPTA, Revista internacional de literatura i cultura medieval i moderna, núm. 14/ desembre 2019/ pp. 276 - 288 ISSN: $2340-4841 \cdot$ doi:10.7203/SCRIPTA.14.16370 


\title{
Dominique de Courcelles. Amour et mort, vie de sainteté dans la littérature et la spiritualité à València au XVème siècle: de saint Vicent Ferrer à Sor Isabel de Villena
}

\begin{abstract}
home més amable e de més afabilitat, més savi, més lleal, més fort, e ab més noble ànimo, ab més virtut e bones costums que tots los altres. E après feren cercar de totes les bèsties qual seria la més bella, més corrent et que pogués sostenir major treball, e qual fos més covinent per a la servitut de l'ome, e de totes, elegiren lo cavall e donaren-lo a l'ome qui fonch elet de mil hòmens hu. E per ço aquel home agué nom cavaller...

E no penses tu que en aquel temps fossen fets cavallers tots aquells que u volien ésser, ans hi foren triats hòmens forts e $\mathrm{ab}$ molta virtut, hòmens leals e piadosos, perquè fossen scut $\mathrm{e}$ defensa de les gents simples, que negú no·ls fes sobres. ${ }^{8}$
\end{abstract}

L'ermite n'hésite pas à rappeler à Tirant que les valeurs guerrières ne sont en rien seulement fondées sur la force : « No pas... emperò cavaller deu tenir virtuts que a altre home no pertanyen».?

C'est ainsi que la valeur spirituelle, religieuse, est la plus haute. Les valeurs guerrières ne sont qu'une condition pour réaliser l'idéal chrétien. C'est la grandeur spirituelle qui détermine l'éclat admirable d'un être humain comme d'un royaume.

L'Eglise, ajoute encore l'ermite, doit être défendue par les chevaliers, parce qu'elle a été établie par Jésus pour prêcher l'Evangile et sanctifier les hommes. C'est ainsi que Tirant, chevalier imaginaire, accomplit à la fin du roman, à la fin de l'histoire, la prouesse de sauver l'empire d'Orient, présentant ainsi au lecteur une métaphore vive de l'absence. Car il n'est plus question depuis longtemps de reconquérir le tombeau vide du Christ à Jérusalem ${ }^{10}$. Il ne faut pas oublier que, dans la dédicace du Tirant, Joanot Martorell avait défini en ces termes le projet du livre: «Noresmenys, a la cavalleria moral donarà lum e representarà los scenacles de bons costums, abolint la textura dels vicis e la ferocitat dels monstruossos actes» ${ }^{11}$.

En s'adressant à ceux qui constituent une chevalerie morale, l'auteur du roman de Tirant sanctionne le glissement politique et moral dont les faits ont établi la nécessité : la quête chevaleresque de Jérusalem échangée en quête de Constantinople se poursuit en quête de chevalerie morale. On a définitivement perdu à partir de 1453 l'empire chrétien d'Orient. C'était bien à un chevalier de València au XVème siècle, quelques décennies avant la fin de la Reconquista de la péninsule Ibérique et peu après la perte de Constantinople, qu'il appartenait de désigner l'ouverture spirituelle de l'histoire, une conversion intérieure.

8Martorell (1992: 53-54).

9 Martorell (1992: 55).

10 De Courcelles (1991: 103-128).

11 Martorell (1992: 2). 
Dominique de Courcelles. Amour et mort, vie de sainteté dans la littérature et la spiritualité à València au XVème siècle: de saint Vicent Ferrer à Sor Isabel de Villena

\section{Ouverture spirituelle d'une histoire au féminin}

Cette ouverture spirituelle de l'histoire, cette conversion intérieure, c'est aussi une femme, l'abbesse des clarisses du couvent de la Trinité de València, Sor Isabel de Villena, en contrepoint du chevalier Joanot Martorell, qui va magnifiquement la signifier, dans la deuxième moitié du XVème siècle, à València.

Contemporaine de Joanot Martorell, Sor Isabel de Villena, fille illégitime de l'écrivain Enrique de Villena et de sang royal, est née en 1430. Dès 1445, elle entre dans le couvent de la Trinité récemment fondé par la reine Marie de Castille, épouse d'Alphonse le Magnanime. Elue abbesse en 1463, elle meurt le 2 juillet 1490. Elle est l'auteur à partir de 1450 d'une très importante Vita Christi en langue valencienne. Sa science et sa sagesse font l'objet de l'admiration de ses contemporains. Miquel Peres, traducteur valencien de l'Imitation de Jésus Christ de Tomàs de Kempis, parue à València en 1482, s'adresse à Sor Isabel dans le prologue en la qualifiant de : «Spill clar en què clarament mirar-se deuen los qui volen entrar en la streta senda de verdadera penitència».

Bernat Fenollar et Pere Martínez dans la dédicace de Lo Passi en cobles, paru à València en 1493, la dénomment «gran doctoressa» et lui demandent conseil.

La première publication de la Vita Christi est réalisée en 1497 à València à la demande de la reine Isabel de Castille et a sans aucun doute beaucoup plus de succès que celle du Tirant, imprimé peu auparavant ; les publications suivantes sont réalisées en 1513 à València avec de nombreuses illustrations et en 1527 à Barcelone. Sor Aldonça de Montsoriu, qui a succédé comme abbesse à Sor Isabel, prend soin de proclamer que cette dernière est bien l'auteur de la Vita Christi publiée à la demande de la reine:

\footnotetext{
E puix ella, humil religiosa, resta lloada d'haver callat lo seu nom en la composición de tan digne llibre, yo en crec atényer no poc mèrit davant Déu en publicar lo nom de tan singular mare, d'immortal memòria digna: sor Isabel de Villena lo ha fet; sor Isabel de Villena l'ha compost; sor Isabel de Villena, ab elegant i dolç stil l'ha ordenat, no solament per a les devotes sors e filles d'obediència que en la tancada casa d'aquest monestir habiten, mas encara per a tots los qui en aquesta breu, enujosa e transitòria vida viuen. ${ }^{12}$
}

Sor Isabel de Villena présente dans son ouvrage une véritable cosmovision dans laquelle l'univers est une manifestation de la divinité ; Dieu se révèle dans l'imperfection des choses visibles de la nature et du monde et par sa grâce qui est l'humanité du Verbe incarné. Création et rédemption sont liées. L'incarnation divine est au centre de l'espace et du temps de la création du monde, dans l'attente d'une seconde venue du Christ qui sera alors le juge suprême pour la parousie. Toute existence humaine, signifie Sor Isabel de Villena, se situe dans le calendrier liturgique qui reprend tout au long de l'année chaque épisode majeur de la vie du Christ et du drame de la rédemption. L'imitation du

12 Isabel de Villena (1995: 64) et 2006. 
Christ est ainsi au centre de la spiritualité chrétienne ; connaître et aimer le Christ revient à posséder l'unique authentique sagesse. Sor Isabel de Villena inscrit ainsi son livre dans la ligne de la célèbre Vita Christi de Landulf de Saxe et également de tout un riche ensemble de textes christocentriques valenciens, et en particulier de la Vida de Jesucrist de Fra Francesc Eiximenis (vers 1330-1409). Il ne faut pas oublier qu'au XVème siècle à València se développe, en lien avec la « devotio moderna » initiée à la fin du XIVème siècle, comme nous l'avons vu plus haut, un certain évangélisme, lié à la nécessité d'une réforme spirituelle basée directement sur la lecture de la Bible. Précisément, la Bible imprimée en 1473 à València, et préparée dès les années 1400 par le chartreux Fra Bonifaci Ferrer, frère de saint Vicent Ferrer, est l'édition princeps de toutes les éditions imprimées dans la péninsule Ibérique ; mais cette Bible de 1473 a été détruite par l’Inquisition.

La savante abbesse cite saint Augustin, saint Jérôme, saint Anselme, saint Bernard et autres auteurs chrétiens, sans jamais les nommer, et en n'hésitant pas à écrire en latin leurs paroles qu'elle juge adéquates à son propos, en les faisant suivre d'une traduction précise ou d'un commentaire. Il est remarquable qu'elle cite également en latin des auteurs de l'Antiquité classique, principalement Sénèque, mais aussi Juvénal, Horace et Ovide. Il est probable qu'elle utilise des florilèges, nombreux à son époque. Mais sans doute connait-elle les traductions qui existent en langue catalane, de même qu'elle connaît assurément la littérature valencienne de son temps.

Sor Isabel propose avec tendresse et réalisme un art de méditer, à partir de personnages imaginés auxquels il est aisé de s'identifier et à partir de situations vraisemblables que l'on peut facilement revivre, bien propre à transformer la réalité quotidienne et à spiritualiser les âmes ${ }^{13}$. L'adaptabilité de son texte est remarquable, la relation entre ses récits et la peinture religieuse de son temps est frappante, comme l'a bien démontré Albert Hauf i Valls à propos de son Speculum Animae ou de la deuxième édition de la Vita Christi faite à València en 1523 et illustrée de nombreuses gravures ${ }^{14}$. Autant et sans doute plus que Joanot Martorell, Sor Isabel, fille de chevalier et de royal lignage, semble bien apte à proposer un art de chevalerie toute céleste, toute spirituelle, pour la rédemption de chaque être humain et pas seulement de l'Occident à travers le renoncement à la reconquête de l'Orient.

La Vita Christi de Sor Isabel de Villena est un art d'aimer, un art d'aimer Dieu, par le moyen de l'imagination et de la représentation épique, héroïque. Le héros paradigmatique est évidemment le « gran cavaller Crist », « soterós », mû par la force de l'amour. Les chevaliers, les héros de cette force de l'amour, à l'imitation du Christ, sont ici essentiellement des femmes : Marie et les vertus qui l'accompagnent, avec toutes les saintes de paradis peuvent sauver l'homme de la chair, du monde et du diable ; elles combattent jusqu'à la mort et en triomphent, obtenant la libération des prisonniers des limbes et établissant un royaume éternel. Il n'est pas impossible que l'abbesse de la Trinité ait

13De Courcelles (2010: 403-418).

14Hauf (1991: 91-124).

SCRIPTA, Revista internacional de literatura i cultura medieval i moderna, núm. 14/ desembre 2019/ pp. 276 - 288 ISSN: 2340-4841 · doi:10.7203/SCRIPTA.14.16370 
Dominique de Courcelles. Amour et mort, vie de sainteté dans la littérature et la spiritualité à València au XVème siècle: de saint Vicent Ferrer à Sor Isabel de Villena

souhaité ici répondre à la misogynie très convenue du Spill de son contemporain valencien Jacme Roig, par ailleurs médecin du monastère et père d’une des moniales de la Trinité.

Pendant que Joanot Martorell part d'un récit antérieur et historique, Sor Isabel s'appuie sur les Evangiles, canoniques et apocryphes. Tous deux utilisent une langue élégante et précise, s'intéressent aux détails, apprécient la courtoisie, les rituels courtois. La cour céleste et spirituelle qui encadre la méditation de Sor Isabel s'inscrit bien en contrepoint de la cour de Constantinople, sensuelle et matérielle, du Tirant. Les bals amoureux du Tirant se transforment dans la Vita Christi en bals merveilleux des anges et des archanges avec les vertus, et c'est ainsi que Sor Isabel exerce une grande influence spirituelle sur les hommes et les femmes de València

\section{Chevaliers et clercs, joutes poétiques et théologiques}

De cette intense créativité littéraire et artistique valencienne, Joan Roís de Corella (vers 1433/14431497), chevalier et maitre en théologie, et Bernat Fenollar (vers 1438-1516), chapelain du roi Ferdinand le Catholique et mathématicien, tous deux admirateurs convaincus de l'abbesse de la Trinité, sont particulièrement représentatifs.

Joan Roís de Corella naît à Gandia, comme Ausias March et probablement aussi Joanot Martorell. Un document de 1471 le dénomme «lo reverend e magnífic Mossèn Joan de Corella, cavaller e mestre en sancta Teología $»^{15}$, ce qui indique qu’il est maitre en théologie, mais sans avoir été ordonné in sacris. En effet, d'autres documents font état de ses enfants Joan Roís de Corella et Estefania Roís de Corella ${ }^{16}$. Il est intéressant par ailleurs de savoir que Joan Roís de Corella prêche fréquemment, par exemple le 4 juillet 1489 dans la cathédrale de València, à l'occasion d'une indulgence concédée par le pape pour la construction de l'autel d'argent de la cathédrale. Bernat Fenollar lui écrit:

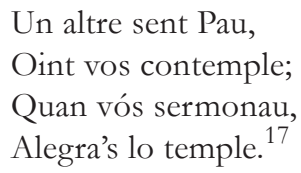

En 1490, Joan Roís de Corella publie à Venise le Psaltiri, «trelladat de latí en romanç per lo reverend mestre Corella », sans doute en raison des suspicions de l'Inquisition valencienne à l'égard des publications de textes bibliques en langue vernaculaire. C'est lui qui prépare pendant plusieurs années la traduction de la vaste œuvre du Cartoixà, chère à la " devotio moderna », qui est publiée

\footnotetext{
15 Riquer (1980: 256).

16 Riquer (1980: 258-259).

17 Roís de Corella (1913: 431).
}

SCRIPTA, Revista internacional de literatura i cultura medieval i moderna, núm. 14/ desembre 2019/ pp. 276 - 288 ISSN: $2340-4841 \cdot$ doi:10.7203/SCRIPTA.14.16370 
Dominique de Courcelles. Amour et mort, vie de sainteté dans la littérature et la spiritualité à València au XVème siècle: de saint Vicent Ferrer à Sor Isabel de Villena

à València en quatre volumes en 1495, 1496, 1500, et que le roi Ferdinand le Catholique réclame dès 1496. Il s'agit de la Vita Christi, écrite par le chartreux Ludolf de Saxe, qui connaît un immense succès.

Comme Sor Isabel de Villena, le chevalier théologien Joan Roís de Corella est attaché à la beauté de la langue, à l'élégance de la prose, à l'harmonie des mots. Qu'elles soient religieuses, romanesques ou mythologiques, ses œuvres déclinent toujours l'amour et la mort sur fond de nécessaire conversion personnelle et spirituelle, qu'il s'agisse, par exemple, de la Història de la gloriosa santa Magdalena, de la Tragèdia de Caldesa ou de la Història de Jason e Medea, basée sur Ovide et Sénèque.

Pour Bernat Fenollar, son ami, chanoine de la cathédrale de València, la poésie doit s'exercer entre poètes en dialogues, en joutes, en débats de toutes sortes. En 1479, Bernat Fenollar est inscrit comme « escrivà de ració » et « capellà e mestre de la capella » du roi Ferdinand le Catholique; en 1510 les jurats de València le nomment titulaire de la «cadira » de mathématiques de l'estudi general. Mais Bernat Fenollar est surtout resté célèbre pour son œuvre poétique et religieuse. Lo Passi en cobles imprimé à València en 1493, dédié à Sor Isabel de Villena, comporte l'histoire versifiée de la Passion «ab algunes altres piadoses contemplacions». Bernat Fenollar et Pere Martines en sont les auteurs; le livre se termine par une Oració de Joan Roís de Corella.

Le «caballer Mossèn» Jaume Gassull, grand ami de Bernat Fenollar, est très actif dans les joutes poétiques. Il écrit en 1474 des louanges en l'honneur de la Vierge et en 1488 il est juge des joutes poétiques en l'honneur de saint Christophe. Il versifie la Història de la gloriosa santa Magdalena de Joan Roís de Corella dans La vida de santa Magdalena en cobles, écrite en 1496 et imprimée à València en 1505 avec de nombreuses images. Il est célèbre par sa participation au Procès de les olives, au Somni de Joan Joan, vers 1496, qui sont des œuvres burlesques et satiriques, parfois obscènes. Plus tard, Gassull glose en castillan le psaume De profundis en l'appliquant à ses «passiones de amon».

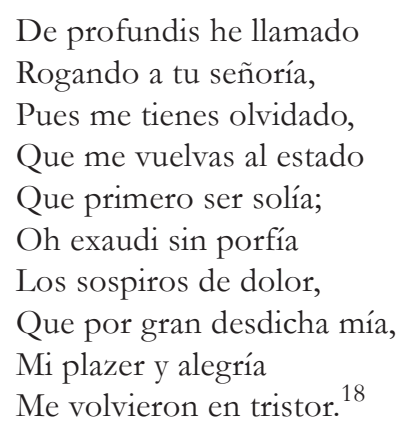

Sainteté et amour sensible, chevalerie et clergie, vie et mort s'entrelacent. L'oubli de la sainteté apporte toujours la douleur.

18 Cancionero General de Hernando del Castillo (1511, f. 203 v). 
Dominique de Courcelles. Amour et mort, vie de sainteté dans la littérature et la spiritualité à

València au XVème siècle: de saint Vicent Ferrer à Sor Isabel de Villena

Or, au début du siècle, Fra Antoni Canals avait insisté dans la mystique Scala de contemplació sur l'importance de la contemplation des goigs ou joies des saints qui permet d'accéder à la vision du Créateur. Le terme goigs -joies- s'applique très tôt, sans doute dès le XIVème siècle, à un genre littéraire particulier qui raconte les goigs des saints pour mieux entraîner à leur suite les fidèles dévots et les faire accéder au paradis à l'heure de la mort. Le XVème siècle est assurément le siècle du développement des goigs, qui prendront toute leur importance dans la vie spirituelle des fidèles après le Concile de Trente $^{19}$. Précisément en 1474, dans un certàmen demeuré fameux, Bernat Fenollar a présenté des goigs commençant par «Los qui·n desijau loar ». On attribue à Bernat Fenollar les goigs de la Verge Maria del Roser, longtemps attribués à saint Vicent Ferrer, qui seront chantés pendant des siècles et jusqu'à aujourd'hui dans toute la Catalogne, València et Baleares ${ }^{20}$. En voici la tornada:

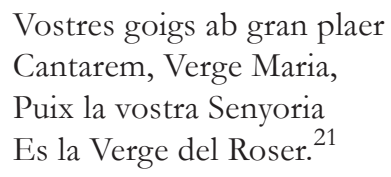

Tout fidèle se trouve ici entraîné par la Vierge vers le séjour divin.

C'est ainsi que València, dans le Royaume de València, au XVème siècle, tient une place majeure dans l'histoire de la littérature et de la spiritualité et dans l'histoire religieuse de la péninsule Ibérique, alors que s'achève la Reconquista et que s'unifient les Espagnes. Chevaliers, saints et saintes, rois et reines, clercs et laïcs, hommes et femmes trouvent ici leurs spirituelles expressions et représentations. Chevalerie et théologie, masculin et féminin se joignent ainsi et se fondent de façon inouie dans l'amour des lettres et des arts. València au XVème siècle est bien un lieu où souffle l'esprit.

19De Courcelles (2008).

20 Ribas (1963) et Mahiques (2011: 37).

21 De Courcelles (2008: 393).

SCRIPTA, Revista internacional de literatura i cultura medieval i moderna, núm. 14/ desembre 2019/ pp. 276 - 288 ISSN: 2340-4841 $\cdot$ doi:10.7203/SCRIPTA.14.16370 
Dominique de Courcelles. Amour et mort, vie de sainteté dans la littérature et la spiritualité à València au XVème siècle: de saint Vicent Ferrer à Sor Isabel de Villena

\section{Bibliografia}

Canals, A. (1975) Scala de Contemplació (introd. et trans. J. Roig Gironella), Barcelona, Fundación Balmesiana.

Cancionero General de Hernando del Castillo (1511), Ed. facsímil de la Real Academia Española, València.

De Courcelles, D. (1991) «Tirant lo Blanc, le meilleur roman du monde » : écriture et impression d'un roman de chevalerie après la disparition de l'empire chrétien d'Orient », Journal of Medieval and Renaissance Studies, 21, pp. 103-128.

—..(2008) La Paraula de l'àngel -una aproximació plural als goigs, Barcelona, Fragmenta Editorial.

- - (2010) «Sor Isabel de Villena dans la Renaissance valencienne: s'écrire soi comme l'autre». Dans L'auteur à la Renaissance (dir. R. Gorris et A. Vanautgaerden), Turnhout, Brepols, pp. 403418.

(2012) «Curial e Güelfa, une voie philosophique et poétique», Estudis lingüistics $i$ culturals sobre Curial e Güelfa, novel-la cavalleresca anònima del segle XV en llengua catalana, New York, John Benjamins Publishing Company, pp. 243-252.

Hauf, A. (1991) «Text i context de l'obra de Sor Isabel de Villena», Literatura valenciana del segle XV -Joanot Martorell i Sor Isabel de Villena, Valencia, Generalitat Valenciana.

Mahiques, J. (2011) «Lo Credo in Deum aplicat per sos articles a la Mare de Déu d'Esperança y los Goigs: un imprés amb obres de Narcís Vinyoles», Miscellania Albert Hauf, PAM, vol. 2.

March, A. (1990) Obra poética completa (Ed. R. Ferreres), Madrid, Clásicos Castalia, vol. 2.

Martorell, J. (1992) Tirant lo Blanch (Ed. A. Hauf), València, Generalitat valenciana, Clàssics Valencians.

Ribas, F. (1963) La data i l'autor dels goigs del Roser (travail inédit, Premi Alfons Bonay i Carbó), Institut d'Estudis Catalans.

Riquer, M. de (1980) Historia de la literatura catalana, Barcelona, Ariel, vol. 3.

Roís de Corella, J. (1991).Obres de J. Roiç de Corella (Ed. R. Miquel i Planas), Barcelona, Biblioteca catalana.

Vicent Ferrer, sant (1971) Sermons (ed. J. Sanchis Sivera), Barcelona, Barcino.

Villena, I. de (1995) Vita Christi (ed. A. Hauf), Barcelona, Ed. 62.

. (2006) Vita Christi (ed. A. Hauf, Ed. facsímil), Biblioteca Valenciana, Generalitat Valenciana, 2006. 